\title{
A pilot study on showcasing the forensic relevance of skin microflora
}

\author{
Toshi Jain \\ State FSL, Sagar \\ Vijay Nema \\ NAARI Pune \\ Mahendra K Gupta \\ Jiwaji University Gwalior \\ R. K Kumawat \\ FSL, Jaipur \\ Pradeep K Singhal \\ RD University Jabalpur \\ Gyaneshwer Chaubey \\ BHU, Varanasi \\ Naveen Kango \\ Dr HS Gour Central University, Sagar
}

PANKAJ SHRIVASTAVA ( $\nabla$ pankaj.shrivastava@rediffmail.com )

State Forensic Science Laboratory https://orcid.org/0000-0002-1647-2390

\section{Research article}

Keywords: Skin Microflora, forensic relevance, 16S rDNA, signature microflora

Posted Date: August 26th, 2020

DOI: https://doi.org/10.21203/rs.3.rs-59353/v1

License: (c) (i) This work is licensed under a Creative Commons Attribution 4.0 International License.

Read Full License 


\section{Abstract \\ Background}

Utility of skin microflora is coming up now with the advancement of molecular techniques and the use of results in predicting infection transmission, genetic relatedness of the source of the origin, forensic use etc. Hence, preparing a catalogue of such endogenous and exogenous microbes would serve as a reference for future studies.

\section{Results}

Isolates identified showed typical pattern of having core microbial communities as found in all the samples and the typical ones which were found only in one individual and not in others. This study showed a potential to identify and classify microbes associated with skin of healthy individuals.

\section{Conclusion}

This can serve as a reference model for future studies and to employ the knowledge into forensic as well as other studies related to health. However, it would be worthwhile to consider similar study with large number of individuals with variable food habits and ethnicity for making an exclusive catalogue for skin microflora.

\section{Background:}

Skin microbiota is an important aspect of our health and routine work. Itsrole in multiple ailments and also in forensic applications is gaining wider grounds now. Our skin harbours both the exogenous or transient microbes due to the environment to which a person is exposed and also the endogenous or the resident microbes residing permanently over the body. Hence, preparing a catalogue of such endogenous and exogenous microbes would serve as a reference for future studies.

Skin microbiota is a diverse and complex community, composition of which is highly dependent on temporal changes, the chemical, biological, and physical surroundings and type of skin[1]. Some genuses of microbes are commensal and are normally found associated with healthy skin. Different species of Staphylococcus are normally found on skin of healthy individuals. At the same time people handling soil etc. also accumulatesa few species of bacillus and pseudomonas from there and these species remain in balanced state with our skin, until they get an opportunity to penetrate into the skin through wounds or cuts. The isolates obtained from each sample were classified using biochemical tests and confirmed further using molecular analysis of 16S rDNA sequencing (Table S2). The obtained results suggested that the individuals working in forensic DNA laboratoryharbouredmore bacterial isolates followed by individuals associated with computers. This finding may also be treated as an alarm towards the health 
and safety measure for forensic DNA experts and computer professionals. Earlier work about microflora associated with computers and mobiles and its forensic use has also endorsed the fact that high and variable number of microbial species remains associated with the keyboards of mobile and laptops, sometimes even depicting the signature microflora of its user [2]. The identified Isolates showed typical pattern of having core microbial communities as found in most of the samples and the typical ones which were found only in one individual and not in others. Interestingly, a few typical profiles were shown by just one or two bacterial species indicative of the profession of the individual from which they were obtained and were not present in others. For instance, individual 5, who worked in agricultural setup showed the presence of Staphylococcus sciuri that is considered as animal bacterial species and is commonly present on skin and mucosal surfaces of a wide range of pets and farm and wild animal[3], whereas Exiguobacterium acetylicum as a typical origin of rhizospheric soil which was found in almost all individuals as they were handling soil either being in their profession or being the homemakers. The isolates obtained before and after spirit wash could distinguish between normal residents and environmental species which temporarily inhabit the skin.

\section{Results:}

We classified the obtained bacterial population into commonly found bacteria on human skin and the ones which were found to be unique in this study as listed in the supplementary Tables3. Multiple species of Staphylococcus were found to be there with an unique one viz. S. vitulinus, while other unrelated bacteria as identified by $16 \mathrm{~S}$ rRNA sequencing were Citrobacterfreundii, Escherichia vulneris, Pseudonocardia, Kocuriarhizophila, Geobacillusstearothermophilus, Bacillus thuringiensis, Aerococcusurinae, Staphylococcus sciuri, Bacillus velezensis, Planococcus, Paenibacillusmucilaginosus, Bacillus pumilus, Cronobactersakazakii, Bacillus tequilensisand Staphylococcus saprophyticus. The presence of these uncommon bacterial genus was correlated with the profession of the individuals and discussed further. The identified bacterial isolates were also analysed by using neighbour joining (NJ) and Principal Component analysis (PCA plot-PAST software v 3.2) methods to see their genetic relatedness as presented in Figure S1 and Figure S2and also through a Table S4.

\section{Discussion:}

Species of Staphylococcus were omnipresent and are well documented to be present on the skin, however $S$. vitulinushas not been reported as skin pathogen and is normally found associated with food and animals, Citrobacter freundii is a pathogen normally found in urinary tract infections and is associated with wound, respiratory, meningitis, and sepsis, Escherichia vulneris is an opportunistic human pathogen, Pseudonocardia is a very typical one that is found associated with the cuticle of leafcutter ants, Kocuriarhizophila has also not been reported from human skin, Geobacillus stearothermophilus is a food contaminant normally found in soil, hot springs, ocean sediment etc., Bacillus thuringiensisis found in agricultural produces,Aerococcusurinae is found in dust of occupied rooms such as grain storage etc., Bacillus velezensis belongs to the Rhizosphere community as like 
Planococcus, Paenibacillus mucilaginosus, Bacillus pumilus, Cronobactersakazakiins an environmental pathogen, whereas Bacillus tequilensis is a plant pathogen. The obtained results also show the individual variation in the microbial profile of individual which is suggestive of use of bacterial profile of individual for forensic purpose (Fig. 1).The tree and PCA plot both have revealed clusters having genetic relatedness and segregated clusters differentiating between bacterial isolates. This classification is a miniaturized version of what all information could be obtained by having an extended study with meticulous observation of all details related to demography and profession of the participating individuals.

\section{Conclusion:}

This study showed a potential to identify and classify microbes associated with skin of healthy individuals. This can serve as a reference for future studies and to employ the knowledge into forensic as well as other studies related to health. However, a comprehensive study with higher number of individuals from all walks of life and with variable food habits and ethnicity may be a better approach for making an exclusive catalogue for skin microflora.

\section{Methods:}

We developed a model study where we collected swabs before and after alcohol wash from hands of 11 healthy individuals having different kind of work profiles and age groups (Table S1). Samples were collected from fingertips of all the individuals using sterile swabs soaked in Phosphate buffered saline(PBS) buffer ( $\mathrm{NaCl} 8 \mathrm{gm}, \mathrm{KCl} 200 \mathrm{mg}, \mathrm{Na}_{2} \mathrm{HPO}_{4} 1.44 \mathrm{gm}, \mathrm{KH}_{2} \mathrm{PO}_{4} 240 \mathrm{mg}$ volume made to 1 litre using distilled water). The samples were obtained before and after a spirit wash to separate endogenous microbes from exogenous ones. The collected swabs were washed in same PBS buffer and were plated on bacteriological media Brain Heart Infusion (BHI) to obtain pure cultures using standard microbiological practices. The cultures were subjected to the gram staining and the battery of biochemical tests for their primary identification. For confirmation of the strains the cultures were subjected to DNA isolation using standard protocol[4] and 16S rDNA PCR using universal 16S rDNA primers viz. 27F-5'-AGAGTTTGATCMTGGCTCAG and 1492R-5'-ACG GCTACCTTGTTACGA on ABI 9700 thermal cycler machine (ThermoFisher Scientific) [5]. The isolates were finally identified by sequencing the 16SrRNA amplicons on ABI 3730xI DNAAnalyzer (Thermo Fisher Scientific, CA, USA) and subsequently matching them with the similar sequences in the database using BLASTN[6].

\section{Abbreviations}

\begin{tabular}{|ll|}
\hline PBS & Phosphate buffered saline \\
\hline BHI & Brain Heart Infusion \\
\hline PCA & Principal Component analysis \\
\hline NJ & Neighbour joining \\
\hline
\end{tabular}




\section{Declarations}

\section{Ethics approval and consent to participate:}

The study was approved by the ethical committee of Jiwaji University, Gwalior wide letter number $\mathrm{JU} / \mathrm{IHEC} / 2017-\mathrm{A} / 06$ dated 9.3.2017. written informed consent was obtained from a parent or guardian for participants under 16 years old along with all other participants of the study.

Consent for publication:

Not applicable

Availability of data and material:

Available on reasonable request.

Competing interests:

Author (S) declares they have no conflict of interest.

\section{Funding:}

There was no funding body for this study.

\section{Authors' contributions:}

PS and MKG planned the study, TJ collected samples, conducted the experiment and did the analysis, RKK helped in the analysis, VN and PS drafted the manuscript, PKS, GC and NK reviewed the manuscript. All the authors read and approved the manuscript.

\section{Acknowledgements:}

Not applicable

\section{References}

1. Bay L, Barnes CJ, Fritz BG, Thorsen J, Restrup MEM, Rasmussen L, et al. Universal Dermal Microbiome in Human Skin. MBio. 2020;11.

2. Fierer N, Lauber CL, Zhou N, McDonald D, Costello EK, Knight R. Forensic identification using skin bacterial communities. Proc Natl Acad Sci. 2010;107:6477-81. 
3. Dakić I, Morrison D, Vuković D, Savić B, Shittu A, Ježek P, et al. Isolation and molecular characterization of Staphylococcus sciuri in the hospital environment. J Clin Microbiol. 2005;43:2782-5.

4. Marmur J. A procedure for the isolation of deoxyribonucleic acid from micro-organisms. J Mol Biol. 1961;3:208-IN1.

5. Nema V. Rectification of artificial molecular recombination with the use of high fidelity enzyme in the amplification of 16S rDNA sequences from Stool sample. All Results Journals Biol. 2012;3:6-9.

6. Altschul SF, Gish W, Miller W, Myers EW, Lipman DJ. Basic local alignment search tool. J Mol Biol. 1990;215:403-10.

\section{Figures}

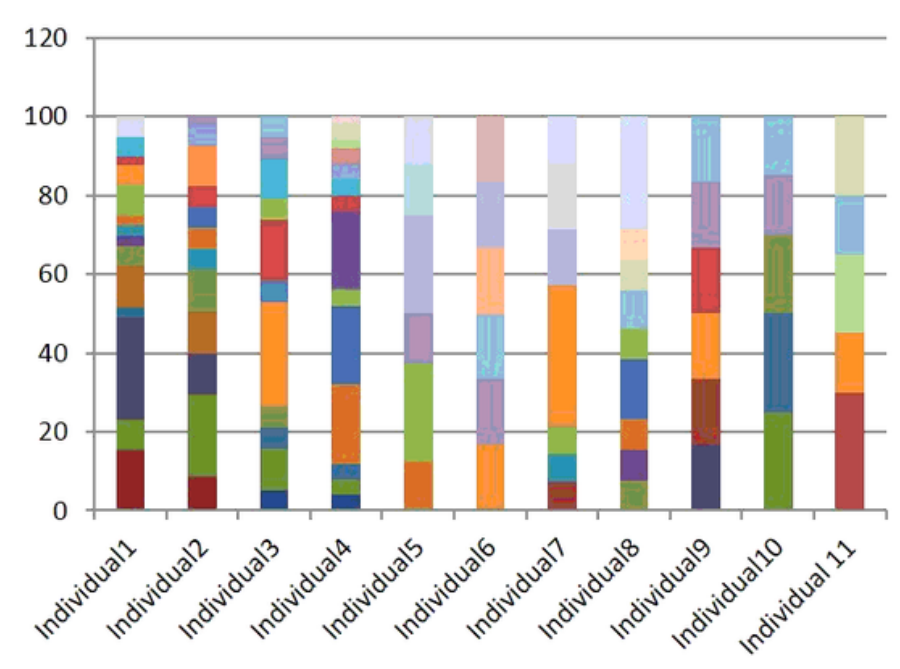

Exiguobacterium mexicanum

Exiguobacterium homiense

Bacillus sonorensis

Bacillus velezensis

Cronobacter sakazakii

Bacillus firmus

Bacillus cereus

- Oceanobacillus iheyensis

Escherichia vulneris

- Corynebacterium striatum

- Kocuria rhizophila

- Pseudonocardia sp.

- Bacillus thuringiensis

- Staphylococcus saprophyticus

- Bacillus subtilis

- Bacillus megaterium

- Staphylococcus pasteuri

- Staphylococcus arlettae

- Staphylococcus lentus

- Staphylococcus warneri
Exiguobacterium profundum

Geobacillus stearothermophilus

Bacillus tequilensis

- Staphylococcus sciuri

- Paenibacillus mucilaginosus strain

- Planococcus sp.

Exiguobacterium acetylicum

Aerococcus urinae

- Citrobacter freundii

- Stenotrophomonas acidaminiphila

- Acinetobacter radioresistens

- Pseudomonas stutzeri

nacillus licheniformis

- Bacillus amyloliquefaciens

- Bacillus pumilus

- Staphylococcus aureus

- Staphylococcus haemolyticus

- Staphylococcus vitulinus

- Staphylococcus epidermidis

- Staphylococcus hominis

\section{Figure 1}

Bar diagram showing the percentage of individual bacterial species from the total bacterial profile of individuals taken in the study 


\section{Supplementary Files}

This is a list of supplementary files associated with this preprint. Click to download.

- TableS4.docx

- TableS3.docx

- Tables2.docx

- Tables1.docx

- Fig.S2.docx

- Fig.S1.docx 